\title{
Detection of Atrial Fibrillation Using a Home Blood Pressure Monitor
}

\section{Thomas Balanis \\ Bernd Sanner}

Internal Medicine, Agaplesion Bethesda Hospital, Wuppertal, Germany
Correspondence: Thomas Balanis Internal Medicine, Agaplesion Bethesda Hospital, Wuppertal, Germany

Email thomasmpalanis@gmail.com
Purpose: Atrial fibrillation (AF) is the most common arrhythmia and is associated with an increased risk of complications. A screening test has the potential to prevent AF-related complications. This study investigated the diagnostic accuracy of an automated device for home blood pressure (BP) monitoring, which implements an algorithm for AF detection.

Patients and Methods: A modified, automated oscillometric device for home BP monitoring (Omron BP785N (HEM-7321-Z), Omron Healthcare) with an AF detector was used to measure the BP in patients. During each BP measurements, the electrocardiogram (ECG) was recorded simultaneously. Simultaneous BP measurements and ECG recordings were obtained from 99 subjects.

Results: Twenty out of 20 patients with atrial fibrillation were correctly recognized by the device and the device correctly identified 67 patients with sinus rhythm as "Not-AF". On the other hand, 12 patients with basic rhythm: sinus rhythm were incorrectly referred to as "atrial fibrillation". In summary, the device has a diagnostic accuracy of $87.88 \%$ with a sensitivity of $100 \%$ and a specificity of $84.8 \%$. On the other hand, in 23 patients, the raw data of the device showed that a body movement occurred during the measurement of the blood pressure. If these subjects were excluded of the analysis, then the diagnostic accuracy of the device would be even better, namely $90.79 \%$. The sensitivity would be $100 \%$ and the specificity $89.5 \%$.

Conclusion: These data suggest that an automated device for home blood pressure has an excellent diagnostic accuracy for detecting an AF and could be used as a reliable screening test for early diagnosis of atrial fibrillation. Body movements have an impact of the accuracy and specificity of a blood pressure monitor.

Keywords: atrial fibrillation detection, blood pressure measurement, Omron BP785N

\section{Introduction}

Atrial fibrillation (AF) is the most common arrhythmia and is a major health care problem. The prevalence increases with age and more than $95 \%$ of the patients with atrial fibrillation are older than 60 years. The prevalence at 80 years is around $10 \%$. The lifetime risk of developing atrial fibrillation is around $25 \%$ for 40 -year-old patients. Atrial fibrillation is somewhat more common in men and in the black population than in women and whites. In addition to age, risk factors include hypertension, diabetes mellitus, heart disease and sleep apnea. Atrial fibrillation is a marker of heart disease and age, so it is difficult to determine to what extent atrial fibrillation itself contributes to morbidity and mortality. Atrial fibrillation is associated with a higher risk of heart failure. The risk of dementia is also increased in atrial fibrillation. ${ }^{1}$ 
Atrial fibrillation is a risk factor of stroke with one in every four strokes occurring as a result of this condition. ${ }^{1}$ As AF is often intermittent, cerebral ischemia is often the initial clinical manifestation of AF. Therefore, screening programs try to solve this important issue. ${ }^{2}$

Hypertension and AF are often concomitant diseases. In the Framingham study, hypertension was a risk factor for $\mathrm{AF}$ by $50 \%$ in men and $40 \%$ in women. ${ }^{3}$ However, because of its higher prevalence in the population, hypertension accounts for more cases of $\mathrm{AF}$ than other risk factors. ${ }^{4,5}$ In the ARIC study (Atherosclerosis Risk in Communities), hypertension was the main contributor to $\mathrm{AF}$, explaining $\approx 20 \%$ of new cases. ${ }^{5}$ Moreover, $60 \%$ to $80 \%$ of the patients with permanent $\mathrm{AF}$ also have arterial hypertension. ${ }^{6}$

Despite the well-established epidemiological association between hypertension and $\mathrm{AF}$, the pathophysiology illustrating why the hypertensive patients develop AF is still not clear. From a clinical point of view, it took several attempts to evaluate the risk of $\mathrm{AF}$ in individual patients. ${ }^{7,8}$ It is not clear whether the risk of AF increases linearly with BP or whether there is a BP threshold above which the risk of this condition definitely increases. ${ }^{9,10}$ Moreover, it is not known if the therapy of BP could reduce the risk of intermittent $\mathrm{AF}$ in hypertensive patients in sinus rhythm. Finally, it is not clear which is the BP threshold in patients with anticoagulation for AF. ${ }^{11}$

Devices for self-monitoring blood pressure by patients at home are used widely in the community in several countries, and many scientific societies have recommended the use of this method for long-term follow-up of treated hypertension. ${ }^{12}$ An algorithm that evaluates the pulse irregularity during BP measurement has been integrated in a home BP monitor. ${ }^{13}$ The first results show that this method could detect AF with good diagnostic accuracy. ${ }^{12,14}$

It is well known that measurement conditions, especially body motion, have a significant influence on the measured blood pressure. ${ }^{15}$ Movement is a major potential source of error for BP measurement and a secondary aim of this study was also to quantify the effect of body movement on BP measurement and detection of atrial fibrillation.

Following the developments in the home blood pressure monitor market, Omron is currently developing an algorithm to obtain and analyze more health-related information from regular blood pressure measurements. The oscillometric technology for home BP measurement is an established method applied by different manufacturers. Omron already includes the Irregular Heart Beat (IHB) detection in most of its products. Now similar products are being made available to the international market, which also claim "AFIB" detection based on analysis of irregularities found in the pressure pulse wave detected in the cuff. In medical practice, diagnosis is now based on EGG readings, which can specify the cause of the irregularities in the pressure pulse wave. The purpose of this study is to test the sensitivity and specificity of the device for the detection of atrial fibrillation as evaluated by simultaneous ECG-recordings. Another objective of the study is to test the accuracy and convenience of the device with a 30 seconds interval compared to an interval of 15 seconds or 60 seconds in the detection of arrhythmia in the general population.

\section{Subjects and Methods Participants}

We examined 101 patients and excluded 2 of them, as they had a pacemaker, in the Department of Internal Medicine of the Agaplesion Bethesda Hospital, Wuppertal, Germany. In all patients' blood pressure measurements with a simultaneous recording of an ECG took place. The study was approved by the Ethics Committee of the Board of Physicians of North Rhine-Westphalia, Düsseldorf, Germany (No. 2019268). Patients were informed about the test examinations and gave their informed consent. The study took place in the ECG Department of the clinic; outpatients and inpatients of the hospital were included. Prior to the examination, the previous illnesses and medication were asked about and documented. The subjects were inpatients and outpatients of the Department of Internal Medicine, they were older than 20 years, including both of female and male gender. Exclusion criteria were as follows: Patients with pacemakers, patients who have a tendency to develop hematomas, patients with shunts for dialysis and patients who cannot maintain a sitting position. During the measurement, the occurrence of body movement should be prevented. Also, patients who are using anticoagulants were excluded due to the risk of developing hematomas and patients who were considered not appropriate by the person in charge.

\section{Device}

An automated oscillometric device for home BP monitoring, which has an additional function that allows $\mathrm{AF}$ 
detection during routine BP measurement, has been developed (BP785N, Omron Healthcare). This device has a TruRead function, which allows setting of interval of three readings in 15,30 or 60 seconds and provides the average value of these three measurements. The automated oscillometric device does not have an AF algorithm ant it detects an IHB only. The device is connected to a tablet recording the pressure pulse wave during the usual BP measurement and by application of an in-built algorithm it analyses the irregularities for episodes of AF. The regular upper arm oscillometric method works by picking up the pressure pulses in the cuff after full occlusion of the brachial artery. When the pressure in the cuff is higher than the blood pressure inside the artery, the artery collapses. The amplitudes of the pulse waves are used to determine the SYS and DIA value, which is displayed on the device. The intervals of the pulse pressure wave are used to determine the pulse rate (heart rate) of the user. The information on the pressure pulse wave can also be used to check the regularity of the heart rate during the measurement. Irregularities can be detected by calculating the average interval and the deviation from each interval to the average value. The irregular ECG signal as in the below tracings will reflect in similar irregularities of the pressure pulse wave in the brachial artery. In the current device, Omron already uses this phenomenon to indicate an irregular heartbeat (IHB) that is detected when there is a deviation of more than $25 \%$ from average. In literature, this has been already related to the presence of atrial fibrillation (Afib) but Omron did not consider this sufficiently supportive evidence for making such claims. In order to improve the reliability of the IHB function to actual arrhythmia further investigation was instigated and a new algorithm was developed. When the same IHB measurement occurs in more than one out of three consecutive measurements the relation between the pulse pressure deviations and actual arrhythmia is much improved.

\section{Procedure}

The medical history, age, weight, arm circumference, medications and a baseline 12-lead ECG were obtained from each participant. Triplicate BP measurements were then taken in the sitting position and with $15 \mathrm{~s}, 30 \mathrm{~s}$, and $60 \mathrm{~s}$ between measurements, using the tested home BP monitor with the AF detector. Simultaneously, the ECG was recorded continuously. The systolic and diastolic BP values and pulse rates measured by the device and the
AF diagnosis carried out (AF, yes/no), were recorded for each measurement. The ECG heart rate and diagnosis of AF or other arrhythmias at baseline, and during each measurement, were made by the investigator. The impact for AF detection was taken into account and not the impact on blood pressure measurement. The protocol was approved by the hospital scientific committee and signed informed consent was obtained from all participants.

\section{Analysis}

The number of participants with atrial fibrillation recognized correctly or incorrectly was documented. In order to determine whether or not an arrhythmia could be detected by the appearance (or non-appearance) of an irregular heartbeat, sensitivity, specificity, positive predictive value, and negative predictive value were determined. As the statistical analysis is concerned, we have used MannWhitney- $U$ test for the parametric values and Fisher's exact test for the non-parametric ones.

\section{Results}

A total of 101 subjects were recruited and we excluded 2 of them. The sample of the patients was heterogeneous. Twenty (20\%) had AF, 79 had (80\%) sinus rhythm (Table 1). The patients showed no difference of convenience between the measurements with an interval of $15 \mathrm{~s}$, $30 \mathrm{~s}$, and 60 seconds. Sixty percent of the patients had arterial hypertension and 20 out of 20 patients with atrial fibrillation had also an arterial hypertension. The average age was 70 years, and the average weight was $74 \mathrm{~kg}$. Furthermore, most of the patients had a polypharmacy and in the subgroup of the patients with atrial fibrillation the average number of drugs was approximately 6 per day. The age and the number of drugs showed a statistically significant difference $(p<0.001)$. The two groups showed no statistical difference regarding the other examined parameters (Table 1).

Twenty-three of the 99 patients had a body movement according to the raw data of the device, but there was no sign of movement in our ECG recordings. According to the instructions in the manual of the device when body movement occurs during a measurement, the arm cuff should be removed and the measurement should be repeated after 2-3 minutes. However, we included those subjects in our study to depict the influence of the body movements on the detection of the atrial fibrillation.

The evaluation of the diagnostic value of the AF detector is presented in Table 2. The ECG showed AF during all BP 
Table I Characteristics of Participants with Atrial Fibrillation and Sinus Rhythm

\begin{tabular}{|c|c|c|c|c|c|}
\hline Characteristics & All Subjects $(n=99)$ & Atrial Fibrillation $(n=20)$ & Sinus Rhythm (n=79) & p value & SD \\
\hline Age (years) & 70.2 & 80.2 & 67.3 & $<0.001$ & 14.88 \\
\hline Weight (kg) & 74.3 & 73.5 & 74.6 & $0.78 \mathrm{I}$ & 11.38 \\
\hline $\operatorname{Arm} \operatorname{circ}(\mathrm{cm})$ & 28.2 & 28 & 28.7 & 0.258 & 2.33 \\
\hline CVD (\%) & 19 & 42 & 18.75 & 0.3581 & - \\
\hline Arterial hypertension (\%) & 60 & 100 & 54 & 0.0003 & - \\
\hline Diabetes mellitus (\%) & 11 & 0 & 11.25 & 0.3389 & - \\
\hline Number of drugs & 3.8 & 6.2 & 3.2 & $<0.001$ & 2.9 \\
\hline Systolic BP (mm Hg) & 138 & 141 & 132 & 0.096 & 20.33 \\
\hline Diastolic BP (mm Hg) & 80 & 79 & 81 & 0.715 & 9.97 \\
\hline
\end{tabular}

Abbreviations: BP, blood pressure; Circ, circumference; CVD, cardiovascular disease; SD, standard deviation.

Table 2 Diagnostic Accuracy of a Home Blood Pressure Monitor to Detect Atrial Fibrillation Without Consideration of the Body Movements Detected by the Device

\begin{tabular}{|l|c|c|}
\hline Statistic & Value & $\mathbf{9 5 \%} \mathbf{C l}$ \\
\hline Sensitivity & $100 \%$ & 83.16 to $100.00 \%$ \\
\hline Specificity & $84.8 \%$ & $74.97 \%$ to $91.90 \%$ \\
\hline Positive predictive value & $62.5 \%$ & $49.74 \%$ to $73.73 \%$ \\
\hline Negative predictive value & $100 \%$ & \\
\hline Accuracy & $87.88 \%$ & $79.78 \%$ to $93.58 \%$ \\
\hline
\end{tabular}

measurements in 20 subjects ( 2 subjects had two measurements) and sinus rhythm during all measurements in 79 subjects. Twenty out of 20 patients with atrial fibrillation were correctly recognized by the device and the device correctly identified 67 patients with sinus rhythm as "Not-AF". On the other hand, 12 patients with basic rhythm: sinus rhythm were incorrectly referred to as "atrial fibrillation". This was due to either premature beats or to a sinus arrhythmia.

On the other hand, in 23 patients, the raw data of the device showed that a body movement occurred during the measurement of the blood pressure. If these subjects were excluded from the analysis, then the diagnostic accuracy of the device would be different and is presented in Table 3. Nine of the 9 patients with atrial fibrillation were correctly recognized by the device and the device correctly identified 60 patients with sinus rhythm as "NotAF". Seven patients with basic rhythm: sinus rhythm was false classified as "atrial fibrillation". The patients showed no difference of convenience between the measurements
Table 3 Diagnostic Accuracy of a Home Blood Pressure Monitor to Detect Atrial Fibrillation with Excluding the Patients with Body Movements During the BP Measurement

\begin{tabular}{|l|c|c|}
\hline Statistic & Value & $\mathbf{9 5 \%} \mathbf{C l}$ \\
\hline Sensitivity & $100 \%$ & 66.37 to $100.00 \%$ \\
\hline Specificity & $89.5 \%$ & $79.65 \%$ to $95.70 \%$ \\
\hline Positive predictive value & $56.25 \%$ & $38.94 \%$ to $72.16 \%$ \\
\hline Negative predictive value & $100 \%$ & \\
\hline Accuracy & $90.79 \%$ & $81.94 \%$ to $96.22 \%$ \\
\hline
\end{tabular}

with an interval of $15 \mathrm{~s}, 30 \mathrm{~s}$, and 60 seconds. Also, there was no difference of the results among the measurements with an interval of $15 \mathrm{~s}, 30 \mathrm{~s}$ and 60 seconds. The current values are based on sample size of 99 subjects and as a result the range of the $95 \%$ confidence interval is relatively high.

\section{Discussion}

This study was conducted in accordance with the Declaration of Helsinki. This study examined the diagnostic accuracy of an automated BP device in detecting AF. In general, the device showed good results with $100 \%$ sensitivity and $85 \%$ specificity. There was no difference either of convenience or of diagnostic accuracy for AF detection between the measurements with time intervals of $15 \mathrm{~s}, 30 \mathrm{~s}$ and $60 \mathrm{~s}$.

Thus far, the diagnosis of AF was carried out with a 12-lead ECG. This method is the gold standard of the diagnosis of AF but a specialist is needed for the 
examination. This is depicted thoroughly in a study that compared the AF diagnosis, made by general practitioners, and a computer software algorithm using a 12-lead ECG, versus a diagnosis made by two cardiologists. ${ }^{16}$ The general practitioners' diagnosis was not good enough with a sensitivity of $80 \%$ and specificity of $92 \%$. The diagnostic value was better when the diagnosis was made together with the computer software, but still not perfect (sensitivity of $92 \%$ and specificity of $91 \%) .{ }^{16}$ So, the conclusion of the authors was that the primary care physicians could not detect AF on ECG in $100 \%$ of the cases despite the help of a computer software. ${ }^{16}$ Munschauer et al investigated the ability of 6203 patients to detect AF with the pulse irregularity. ${ }^{17}$ Unfortunately, $27 \%$ of the patients could not feel their pulse, and of those who did, 9\% could not tell whether it was irregular. ${ }^{17}$

Due to the wide usage of devices for home BP measurement in the community, the conception that such devices could also have specific algorithms that are able to detect arrhythmias is inspiring. Early algorithms that could detect an irregularity in the pulse were integrated in home BP devices could not diagnose an $\mathrm{AF}$ from other arrhythmias. Therefore, their specificity for AF diagnosis is extremely low. Wiesel et al developed an algorithm for $\mathrm{AF}$ detection during routine $\mathrm{BP}$ measurement, which has been integrated in the home BP monitor. They tested the diagnostic value of an AF-detecting home BP device (modified Omron 712C) by 450 subjects, of whom 54 had AF on ECG. ${ }^{13}$ In this study, the sensitivity was $100 \%$, the specificity $91 \%$, and the diagnostic accuracy $92 \%$ for detecting AF. ${ }^{13}$ Another study by Wiesel et $\mathrm{al}^{13}$ assessed the same in 205 people, of whom 52 had AF on ECG. The sensitivity and specificity of a single measurement for detecting AF was $98 \%$ and $88 \%$, respectively. For triplicate measurements, the diagnostic accuracy was improved (sensitivity, 100\%; specificity, $89 \%$ ). These results are similar to the findings of our new study (Table 2). In comparison with the above-mentioned studies, our study's benefit is that we achieved high accuracy and same level despite that our algorithm is much simpler.

The main issues of our new study are that all BP measurements were performed together with continuous ECG recording and the design of the study allowed the detection of changes in rhythm during the triplicate BP measurements. In addition, the device was tested in difficult diagnostic conditions, because some patients had non-AF arrhythmias. We supposed that a false-positive diagnosis of AF might be frequent in these patients. Such a weakness (low specificity) of this test would often cause a "false alarm". Attractively, even in these complicated circumstances, the device showed a good diagnostic value. In agreement with the results of Wiesel et al and after comparing single, duplicate and triplicate measurements, these results demonstrated that triplicate measurements are the best approach in terms of diagnostic accuracy. ${ }^{14}$ Also, this screening method seems to have a better diagnostic value than the general practitioners' diagnosis carried out by ECG along with a software. ${ }^{16}$ Nevertheless, that we performed triplicate measurements, the device over diagnosed AF in 12 patients. All these subjects had premature extra beats, which were concerned as clinically irrelevant arrhythmias. In our opinion, even in these cases, the patients might benefit from a medical consultation because we cannot distinguish a clinically relevant arrhythmia from an irrelevant one without carrying out an ECG. A limitation from the integrated algorithm is that it cannot detect an atrial flutter.

Another important feature is the high negative predictive value of the device (98\%). This is an important feature because some patients would have visited their physician because of palpitations due to stress or another reason. Visiting their physician may be inconvenient and add some cost. The fear of these patients about having an AF could be reduced using this screening method.

There is a group of patients that would gain from using this device. Because the device is designed to detect AF, it would be reasonable to use this device in patients with risk factors for a cerebral embolism. The majority of our subjects in our study had hypertension, diabetes mellitus, congestive heart failure, or aged $\geq 65$ years, which are risk factors for a stroke. Therefore, the high sensitivity of this device is very useful in these highrisk patients. In addition, long-term studies following patients using this device at home need to be carried out to determine the number of new episodes of AF detected and the cost of the false-positive readings.

Furthermore, automated BP monitors give accurate BP measurements in patients with $\mathrm{AF}$ and that can be applied in everyday clinical practice. ${ }^{18,19}$ This issue deserves special attention.

The limitation for our device includes potential influences of premature heart beats and of artifacts. They could influence the diagnostic accuracy that results in false-positive results. It also cannot be excluded that the diagnostic performance of device may even be more limited in an everyday clinical practice ambulatory where movement artifacts could be more frequent. According to current guidelines, final confirmation of AF still remains an ECG-based diagnosis. ${ }^{1}$ Thus, BP-based AF detection devices should be evaluated only as a screening tool with a need for an ECG and not for diagnosing an AF. Moreover, a frequent problem in the clinical practice is that 
AF is often asymptomatic and the diagnosis of AF is made unfortunately after the first thromboembolic event occurs. Higher detection rates and earlier identification of unknown AF could contribute to reduce the risk of stroke and other consequences by initiating appropriate therapeutic measures. ${ }^{1}$ Extended screening methods have been shown to have better detection rates. Therefore, the use of rhythm-recording devices, such as Holter electrocardiography (ECG) monitors or loop recorders, ${ }^{20,21}$ was associated with a more than fivefold higher incidence of AF after 30-day screening inpatients with cryptogenic stroke in the EMBRACE (30-Day Cardiac Event Monitor Belt for Recording Atrial Fibrillation After a Cerebral Ischemic Event) trial. ${ }^{21}$ Hence, the usual measurement of pressure once or twice a day may not adequately detect intermittent atrial fibrillation.

Thus, the smartphone applications that enable rhythm analysis are very promising. They have been shown to detect atrial fibrillation with high diagnostic accuracy. ${ }^{22}$ Professor Dörr et al were able to demonstrate that smartwatches offer a good option to comfortably and relatively easily monitor the heart rhythm. In a prospective controlled study, the study group assessed the ability of a smartwatch to detect an atrial fibrillation in 508 subjects with and without atrial fibrillation. The recordings by the smartwatches were analyzed using an automated algorithm regarding atrial fibrillation. The results were compared to a mobile electrocardiogram (ECG). These ECGs were afterwards assessed by cardiologists who did not know the history of the participants. This study demonstrated that the smartwatch could detect atrial fibrillation as good and as the mobile ECG. ${ }^{22}$ Using such technology could potentially upgrade rhythm screening to the next level as compared to previous approaches with other devices by making convenient long-term screening or extended noninvasive rhythm analysis possible in individuals who are not suitable for other screening methods. ${ }^{23}$ This method along with other technologies has recently been added to the AF screening recommendations of the European Heart Rhythm Association. ${ }^{24}$ A recently published study suggests that smartwatches (for instance, Apple Watch) are useful for detecting rhythm abnormalities like atrial fibrillation, but there is still a long way to go before they can be trusted for clinical management. ${ }^{25}$ Researchers at Cleveland Clinic studied 50 postoperative cardiac surgery patients in an attempt to determine the utility of the Apple Watch 4 - a hightech smartwatch with ECG features - in detecting atrial fibrillation. Looking only at what the Apple Watch displays on its 40-millimeter screen, Apple's software identified only 34 of 90 instances, namely $41 \%$ of the total. The display also identified Afib in 19 of 25 patients who experienced an arrhythmia more than once, but reviewing the display data in conjunction with Apple's retrievable PDF of waveform strips resulted in improved identification. The device correctly identified $96 \%$ of the instances of atrial fibrillation in users, and it identified Afib in 24 of the 25 patients who experienced more than one episode. In patients whose hearts were in normal sinus rhythm, the Apple Watch did not send out any notifications of Afib, indicating that there were not any false positives. ${ }^{25}$ These data suggest that the $41 \%$ sensitivity of the Apple Watch display is much lower in comparison with our study. This can be due to a probable insufficient signal quality due to movements and artifacts. We already showed that the body movement has a significant influence on the diagnostic accuracy. Hence, it needs some more improvement before these wearables can be fully incorporated into current clinical management. Moreover, our study device is an automated oscillometric device for home BP monitoring, so that the patients can have reliable BP measurements. On the other hand, in the apple heart study, among patients with sinus rhythm, none were designated as Afib, in other words, no false-positive results. This comes in contrast to our study, where we had falsepositive results due to supraventricular or ventricular extrasystoles. As a result, an improvement of the algorithm of detection of atrial fibrillation in this field of the premature extra beats is necessary.

Another important factor that this study points out is that the detection of atrial fibrillation during the BP measurement is also affected by external stimuli, such as patient motion. It is obvious that the diagnostic accuracy would be much lower if the patient would not stay still. In line with the instructions, the occurrence of body movement should be prevented, and in case the display shows the icon, the measurement should be repeated after rest of 2-3 minutes. Therefore, we included both results in the final report to indicate the importance of adherence to the instructions for use. This will contribute to the awareness of proper measurement of blood pressure using oscillometric devices.

In conclusion, this study depicted that a home BP monitor with an integrated algorithm for AF diagnosis appears to have a very good diagnostic accuracy. The widespread usage of this algorithm in automated home $\mathrm{BP}$ in the community appears to be an excellent screening test. Of course, its ability to detect intermittent AF requires further investigation. Subjects with $\mathrm{AF}$, detected by the device, should consult their physician as soon as possible in order to confirm the AF diagnosis by ECG before any therapeutical intervention is made. Home monitoring of blood pressure for patients with hypertension has been shown to be beneficial. Since hypertension is the most 
common risk factor associated with $\mathrm{AF}$, the use of a home blood pressure monitors to detect asymptomatic AF could provide additional value. This blood pressure monitor with the new algorithm has a high sensitivity and is able to correctly determine the majority of AF rhythms. This algorithm has been implemented in the newest series of blood pressure monitors from Omron, with the Afib mode.

\section{Abbreviations}

$\mathrm{AF}$, atrial fibrillation; BP, blood pressure; CI, confidential interval; Circ, circumference; cm, centimeters; CVD, cardiovascular disease; DIA, diastolic; ECG, electrocardiogram; IHB, irregular heart beat; kg, kilograms; Not-AF, not atrial fibrillation; SD, standard deviation; SYS, systolic.

\section{Disclosure}

Mr Thomas Balanis reports grants from Omron, during the conduct of the study; Prof. Dr Bernd Sanner reports grants from Omron, during the conduct of the study; personal fees from speaker fees, outside the submitted work. The authors declare no other conflicts of interest in this work.

\section{References}

1. Kirchhof P, Benussi S, Kotecha D, et al. ESC guidelines for the management of atrial fibrillation developed in collaboration with EACTS. Eur Heart J. 2016;37(38):2893-2962.

2. Fitzmaurice DA, Hobbs FD, Jowett $S$, et al. Screening versus routine practice in detection of atrial fibrillation in patients aged 65 or over: cluster randomised controlled trial. BMJ. 2007;335:383-386. doi:10.1136/bmj.39280.660567.55

3. Benjamin EJ, Levy D, Vaziri SM, et al. Independent risk factors for atrial fibrillation in a population-based cohort. The Framingham Heart study. JAMA. 1994;271:840-844. doi:10.1001/ jama.1994.03510350050036

4. Lau YF, Yiu KH, Siu CW, et al. Hypertension and atrial fibrillation: epidemiology, pathophysiology and therapeutic implications. J Hum Hypertens. 2012;26:563-569. doi:10.1038/jhh.2011.105

5. Huxley RR, Lopez FL, Folsom AR, et al. Absolute and attributable risks of atrial fibrillation in relation to optimal and borderline risk factors: the Atherosclerosis Risk in Communities (ARIC) study. Circulation. 2011;123:1501-1508. doi:10.1161/CIRCULATIONAHA.110.009035

6. Nabauer M, Gerth A, Limbourg T, et al. The Registry of the German Competence NETwork on Atrial Fibrillation: patient characteristics and initial management. Europace. 2009;11:423-434. doi:10.1093/ europace/eun369

7. Benjamin EJ, Chen PS, Bild DE. Prevention of atrial fibrillation: report from a national heart, lung, and blood institute workshop. Circulation. 2009;119:606-618. doi:10.1161/CIRCULATIONAH A. 108.825380

8. Alves-Cabratosa L, García-Gil M, Comas-Cufí M, et al. Incident atrial fibrillation hazard in hypertensive population: a risk function from and for clinical practice. Hypertension. 2015;65:1180-1186. doi:10.1161/ HYPERTENSIONAHA.115.05198
9. Verdecchia P, Mazzotta G, Angeli F, et al. Above which blood pressure level does the risk of atrial fibrillation increase? Hypertension. 2012;59:184-185. doi:10.1161/HYPERTENSIONAHA.111.187260

10. Rabkin SW, Moe G. The case against using hypertension as the only criterion for oral anticoagulation in atrial fibrillation. Can J Cardiol. 2015;31:576-579. doi:10.1016/j.cjca.2015.03.022

11. Manolis AJ, Rosei EA, Coca A, et al. Hypertension and atrial fibrillation: diagnostic approach, prevention and treatment. J Hypertens. 2012;30:239-252. doi:10.1097/HJH.0b013e32834f03bf

12. Stergiou GS, Karpettas N, Protogerou A, et al. Diagnostic accuracy of a home blood pressure monitor to detect atrial fibrillation. $J$ Hum Hypertens. 2009;23:654-658. doi:10.1038/jhh.2009.5

13. Wiesel J, Wiesel D, Suri R, et al. The use of a modified sphygmomanometer to detect atrial fibrillation in outpatients. Pacing Clin Electrophysiol. 2004;27:639-643. doi:10.1111/j.1540-8159.2004.00499.x

14. Wiesel J, Herschman Y, Messinao FC. Detection of atrial fibrillation using a Microlife automatic blood pressure monitor (abstract). J Clin Hypertens. 2008;10(suppl A):A84-A85.

15. Zheng D, Giovannini R, Murray A. Effect of respiration, talking and small body movements on blood pressure measurement. J Hum Hypertens. 2012;26:458-462. doi:10.1038/jhh.2011.53

16. Mant J, Fitzmaurice DA, Hobbs FD, et al. Accuracy of diagnosing atrial fibrillation on electrocardiogram by primary care practitioners and interpretative diagnostic software: analysis of data from screening for atrial fibrillation in the elderly (SAFE) trial. $B M J$. 2007;335:380-385. doi:10.1136/bmj.39227.551713.AE

17. Munschauer FE, Sohocki D, Carrow SS, et al. A community education program on atrial fibrillation: implications of pulse self-examination on awareness and behavior. J Stroke Cerebrovasc Dis. 2004;13:208-213. doi:10.1016/j.jstrokecerebrovasdis.2004.08.001

18. Jani B, Bulpitt CJ, Rajkumar C. The accuracy of blood pressure measurement in atrial fibrillation. $J$ Hum Hypertens. 2006;20:543-545. doi:10.1038/sj.jhh.1002016

19. Watson T, Lip GY. Blood pressure measurement in atrial fibrillation: goodbye mercury. J Hum Hypertens. 2006;20:638-640. doi:10.1038/ sj.jhh.1002031

20. Wachter R, Groschel K, Gelbrich G, et al. Holter-electrocardiogrammonitoring in patients with acute ischemic stroke (Find-AFRANDOMISED): an open-label randomised controlled trial. Lancet Neurol. 2017;16:282-290. doi:10.1016/S1474-4422(17) 30002-9

21. Gladstone DJ, Spring M, Dorian P, et al. Atrial fibrillation in patients with cryptogenic stroke. $N$ Engl J Med. 2014;370:2467-2477. doi:10.1056/NEJMoa1311376

22. Dörr M, Nohturfft V, Brasier N, et al. The WATCH AF trial: smart WATCHes for detection of atrial fibrillation. JACC Clin Electrophysiol. 2019;5(2):199-208. doi:10.1016/j.jacep.2018.10.006

23. Carpenter A, Frontera A. Smart-watches: a potential challenger to the implantable loop recorder? EP Europace. 2016;18(6):791-793. doi:10.1093/europace/euv427

24. Mairesse G, Moran P, Van Gelder I, et al. Screening for atrial fibrillation: a European Heart Rhythm Association (EHRA) consensus document endorsed by the Heart Rhythm Society (HRS), Asia Pacific Heart Rhythm Society (APHRS), and Sociedad Latinoamericana de Estimulación Cardíaca y Electrofisiología (SOLAECE). EP Europace. 2017;19(10):1589-1623. doi:10.1093/ europace/eux 177

25. Seshadri D, Bittel B, Browsky D, et al. Accuracy of apple watch for detection of atrial fibrillation. Circulation. 2020;141:702-703. doi:10.1161/CIRCULATIONAHA.119.044126 


\section{Publish your work in this journal}

Vascular Health and Risk Management is an international, peerreviewed journal of therapeutics and risk management, focusing on concise rapid reporting of clinical studies on the processes involved in the maintenance of vascular health; the monitoring, prevention and treatment of vascular disease and its sequelae; and the involvement of metabolic disorders, particularly diabetes. This journal is indexed on PubMed Central and MedLine. The manuscript management system is completely online and includes a very quick and fair peerreview system, which is all easy to use. Visit http://www.dovepress. com/testimonials.php to read real quotes from published authors 